\title{
Pigs are not susceptible to SARS-CoV-2 infection but are a model for viral immunogenicity studies
}

\author{
Júlia Vergara-Alert ${ }^{1,2}$ (D) | Jordi Rodon ${ }^{1}$ | Jorge Carrillo ${ }^{3}$ | Nigeer $\mathrm{Te}^{1}$ | Nuria Izquierdo- \\ Useros $^{3,4}$ | María Luisa Rodríguez de la Concepción ${ }^{3}$ | Carlos Ávila-Nieto ${ }^{3}$ | \\ Víctor Guallar $^{5,6}$ | Alfonso Valencia ${ }^{5,6}$ | Guillermo Cantero ${ }^{1}$ | Julià Blanco ${ }^{3,4,7}$ | \\ Bonaventura Clotet $^{3,4,7}$ | Albert Bensaid ${ }^{1,2}$ | Joaquim Segalés ${ }^{2,8,9}$ (D)
}

${ }^{1}$ IRTA, Centre de Recerca en Sanitat Animal (CReSA, IRTA-UAB), Campus de la Universitat Autònoma de Barcelona, Barcelona, Spain

${ }^{2}$ OIE Collaborating Centre for the Research and Control of Emerging and Re-emerging Swine Diseases in Europe (IRTA-CReSA), Barcelona, Spain

${ }^{3}$ IrsiCaixa AIDS Research Institute, Badalona, Spain

${ }^{4}$ Germans Trias i Pujol Research Institute (IGTP), Badalona, Spain

${ }^{5}$ Barcelona Supercomputing Center (BSC), Barcelona, Spain

${ }^{6}$ Catalan Institution for Research and Advanced Studies (ICREA), Barcelona, Spain

${ }^{7}$ University of Vic-Central University of Catalonia (UVic-UCC), Vic, Spain

${ }^{8} \mathrm{UAB}$, Centre de Recerca en Sanitat Animal (CReSA, IRTA-UAB), Campus de la Universitat Autònoma de Barcelona, Barcelona, Spain

${ }^{9}$ Departament de Sanitat i Anatomia Animals, Facultat de Veterinària, UAB, Barcelona, Spain

\section{Correspondence}

Júlia Vergara-Alert and Joaquim Segalés, Centre de Recerca en Sanitat Animal (CReSA), Institut de Recerca i Tecnologia Agroalimentàries (IRTA), Edifici CReSA, Campus UAB, 08193 Bellaterra, Barcelona, Spain.

Emails: julia.vergara@irta.cat (J. V.-A.); joaquim.segales@irta.cat (J.S.)

Funding information

Grifols Pharmaceutical

\begin{abstract}
Conventional piglets were inoculated with severe acute respiratory syndrome coronavirus 2 (SARS-CoV-2) through different routes, including intranasal, intratracheal, intramuscular and intravenous ones. Although piglets were not susceptible to SARSCoV-2 and lacked lesions or viral RNA in tissues/swabs, seroconversion was observed in pigs inoculated parenterally (intramuscularly or intravenously).
\end{abstract}

\section{KEYWORDS}

immunogenicity model, inoculation routes, lack of susceptibility, pig, SARS-CoV-2 
The coronavirus disease 2019 (COVID-19) is an infectious disease that has caused a global pandemic with more than 36 million infected people from around 200 countries or territories, with more than 1 million deaths to date (World Health Organization (WHO) 2020). The causative agent of COVID-19, the severe acute respiratory syndrome coronavirus 2 (SARS-CoV-2), is assumed to be originated in bats, since the bat-borne coronavirus RaTG13 is the closest genetic relative to date (Andersen et al., 2020; Zhou et al., 2020). Several species have been studied to determine their potential role as intermediate hosts (Shi et al., 2020). Moreover, animal models to recapitulate a COVID-19-like disease are considered a major research line and required for the development of therapeutic drugs and prophylactic compounds.

Besides several modelling studies proposing potential animal species susceptible to SARS-CoV-2 (Damas et al., 2020; Qiu et al., 2020; Veljkovic et al., 2020), multiple experimental infections have already shown a broad range of susceptible animals. Specifically, Egyptian fruit bat, ferret, golden Syrian hamster cat, mice expressing humanized angiotensin-converting enzyme 2 (ACE2), BALB/c mice (using a mutated SARS-CoV-2 by several cell culture passages) and some non-human primate species are permissive to viral infection, developing from subclinical to mild-to-moderate respiratory disease (Bao et al., 2020; Halfmann et al., 2020; Kim et al., 2020; Rockx et al., 2020; Shi et al., 2020; Yu et al., 2020). From an experimental point of view, dog susceptibility to SARS-CoV-2 is limited, since inoculated animals can partly seroconvert (Shi et al., 2020). In contrast, the intranasal inoculation of chicken, duck and pig resulted in no evidence of infection (Schlottau et al., 2020; Shi et al., 2020).

Pig is commonly used in research because of the similarities existing with humans in terms of anatomy, genetics, physiology and, also, immunology. Indeed, experiments in pigs are likely to be more predictive of therapeutic and preventive treatments in humans than experiments in rodents (Meurens et al., 2012). However, since pigs are not susceptible to SARS-CoV-2 infection when inoculated intranasally (Schlottau et al., 2020; Shi et al., 2020), the possibility to develop a swine infection model with this virus using other potential inoculation routes deserves investigation. The main rational to test pigs is that the ACE2 receptor of this species is functional either by transfecting swine ACE2 in HeLa cells (which do not express constitutively the human ACE2) (Zhou et al., 2020) or that pseudoparticles with the S protein of SARS-CoV-2 are able to infect swine kidney cells (Letko et al., 2020). Furthermore, the ACE2 protein is expressed in all major tissues of pigs as assessed by immunohistochemistry (Xiao et al., 2020). In consequence, to set up a putative COVID-19 pig model, we investigated the effect of different natural and non-natural routes of SARS-CoV-2 inoculation in domestic pigs (Sus scrofa domesticus).

For the purpose, four groups of five 5- to 6-week-old conventional piglets (Landrace $\times$ Large White) were selected and inoculated by means of different routes: intranasal (IN, $1.5 \mathrm{ml} /$ nostril; total volume of $3 \mathrm{ml}$ ), intratracheal (IT, $3 \mathrm{ml}$ ) as previously described (GarciaMorante et al., 2016), intramuscular (IM, $1 \mathrm{ml}$ in each side of the neck muscles; total volume $2 \mathrm{ml}$ ) or intravenous (IV, $2 \mathrm{ml}$ ), with a final dose of $10^{5.8}$ tissue culture infectious dose $\left(\mathrm{TCID}_{50}\right)$ of the SARS-CoV-2 isolate (GISAID ID EPI_ISL_510689) per each animal. The IT and IV groups were anaesthetized with $10 \mathrm{mg} / \mathrm{kg}$ of ketamine and $0.8 \mathrm{mg} /$ $\mathrm{kg}$ xylazine prior inoculation. A passage-2 SARS-CoV-2 was propagated and titrated in Vero E6 cells (ATCC CRL-1586), following the same protocol as for other coronaviruses (Rodon et al., 2019). Two extra pigs were used as negative controls.

All animals were seropositive against porcine respiratory coronavirus, as determined by a commercial ELISA (INgezim Corona Diferencial 2.0 [TGEV/PRCV]). Taking into account that no antibody cross-reactivity between alpha- and beta-coronaviruses has been described (Okba et al., 2020), the animals were kept into the study. Initial reactivity against PRCV was expected since this virus is ubiquitous in the European swine livestock (Saif et al., 2012; Vidal et al., 2019).

Animal experiments were approved by the Institutional Animal Welfare Committee of the Institut de Recerca i Tecnologia Agroalimentàries (CEEA-IRTA) and by the Ethical Commission of Animal Experimentation of the Autonomous Government of Catalonia and conducted by certified staff. Experiments with SARS-CoV-2 were performed at the Biosafety Level-3 (BSL-3) facilities of the Biocontainment Unit of IRTA-CReSA (Barcelona, Spain).

On 2 and 22 days post-inoculation (dpi), two and three animals/group (IT, IM and IV), respectively, were euthanized. Since IN inoculation was already demonstrated as non-effective to cause SARS-CoV-2 infection (Shi et al., 2020), pigs inoculated by this route were euthanized on days 1 and 2 pi to assess evidence of a possible transient early infection in tissues. Negative control animals were euthanized prior to the start of the experiment. Samples were collected and processed as previously described (Vergara-Alert et al., 2017). Briefly, complete necropsies were performed in all animals. Several tissues (frontal, medial and caudal turbinates; proximal, medial and distal trachea; large and small bronchus, left cranial, mediodorsal and caudal lung areas; kidney; liver; heart; and spleen) were taken, fixed by immersion in $10 \%$ neutral-buffered formalin, embedded in paraffin and sectioned at $3 \mu \mathrm{m}$ to prepare slides. Histology slides were stained with haematoxylin and eosin (HE) to assess potential microscopic lesions. Besides, the same tissues plus ileum, cervical lymph node (LN), mediastinal LN, mesenteric LN, olfactory bulb, tonsil, thymus, parotid salivary gland, adrenal, pancreas, brainstem, eyelids and bone marrow were also taken in Dulbecco's modified Eagle medium (DMEM) in tubes with beads to perform SARS-CoV-2 upE gene detection by RT-qPCR (Corman et al., 2020). Nasal and rectal swabs were also taken (daily during the first week and at 14 and $22 \mathrm{dpi}$ ) to analyse them for the presence of viral RNA by means of the above-mentioned RT-qPCR. Serum samples collected on days 0, 14 and 22 pi were tested for the presence of antibodies against SARSCoV-2 spike S1 + S2 and nucleocapsid (N) proteins by in-house ELISAs (Institut de Recerca de la sida (Irsicaixa), 2020). Also, a 
FIGURE 1 (a) Antibody detection of pigs experimentally inoculated with SARSCoV-2. Detection of antibodies against the spike protein (days 0, 14 and 22 pi) by ELISA in sera from animals inoculated intratracheally (No. 8-10, IT group), intramuscularly (No. 13-15, IM group) and intravenously (No. 18-20, IV group). (b) Antibody detection of pigs experimentally inoculated with SARS-CoV-2. Detection of neutralizing antibodies (days 0 and $22 \mathrm{pi}$ ) in sera from animals inoculated intratracheally (No. 8-10, IT group), intramuscularly (No. 13-15, IM group) and intravenously (No. 18-20, IV group). The graph shows the reciprocal serum dilution showing neutralization activity versus dpi. Dotted line indicates limit of detection of the assay ( $1 / 20$ serum dilution). A value of 5 was assigned to undetectable neutralization activity. Unpaired Student's $t$ tests were performed to assess whether neutralizing antibodies significantly increased at $22 \mathrm{dpi}$. ns, not significant; ${ }^{*} p$-value $<.05$
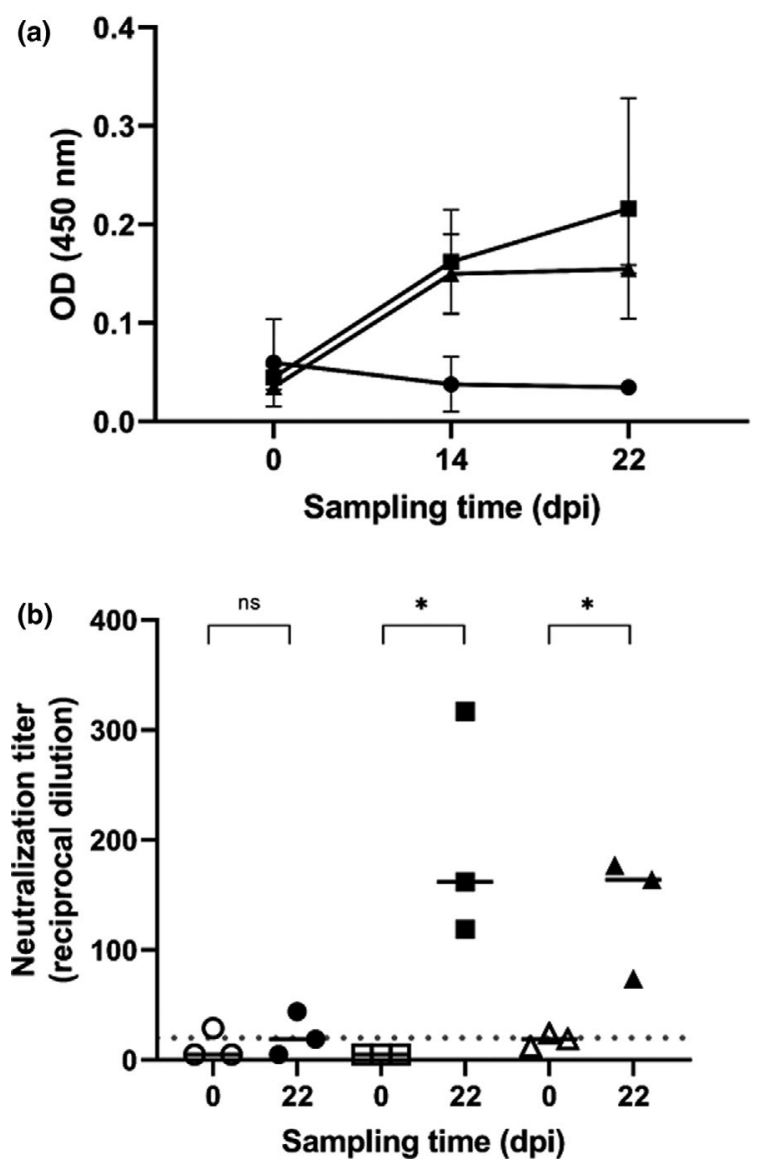

O- PIGS 8-10, IT group

प. PIGS 13-15, IM group

$\Delta$ A PIGS 18-20, IV group virus neutralization assay was performed following a previous protocol with a minor modification (Rodon et al., 2020), the serial dilutions of sera and SARS-CoV-2 were incubated for $1 \mathrm{hr}$ at $37^{\circ} \mathrm{C}$ prior the plate assay performance.

All animals were daily monitored but none of them showed clinical signs after SARS-CoV-2 inoculation. Also, no gross or microscopic lesions attributable to SARS-CoV-2 infection were found in any of the studied animals from all inoculation groups as well as control ones (data not shown).

None of the pigs had nasal or rectal shedding of viral RNA. Proximal trachea from one $\mathrm{IN}$-inoculated animal was positive at $1 \mathrm{dpi}$ for viral RNA ( $\mathrm{Cq}=24.36)$. The remaining tissues from this animal and the rest of pigs resulted negative for RT-qPCR (qPCR detection limit of 38.6 cycles).

By 14 and 22 dpi, low levels of antibodies directed against the Spike protein could be detected in all animals from IM and IV groups (Figure 1a). Furthermore, these pigs also showed neutralizing antibody titres at $22 \mathrm{dpi}$ (ranging from 74 to $317 \mathrm{SNT}_{50}$ reciprocal dilution titre) (Figure $1 \mathrm{~b}$ ). Also, low antibody levels targeting the $\mathrm{N}$ protein were found in one out of three IM and all IV inoculated animals by the end of the experiment (data not shown). Importantly, one single animal from the IT group did not show antibodies against the $\mathrm{S}$ but had antibodies against the $\mathrm{N}$ protein as well as neutralizing titres $\left(\mathrm{SNT}_{50}\right.$ reciprocal dilution titre of 29) at day $0 \mathrm{pi}$, which might suggest a potential cross-reaction with another coronavirus infecting swine. Of note, these antibodies against the $\mathrm{N}$ protein decreased by the time the experiment finished, suggesting they were of maternal origin. In addition, this animal did not show seroneutralizing antibodies at the $22 \mathrm{dpi}$ (Figure 1b).

Present data indicate that SARS-CoV-2 was not able to infect pigs by any of the tested routes, namely IN, IT, IM and IV. Therefore, our efforts confirm earlier experiments indicating lack of susceptibility of infection by the pig (Schlottau et al., 2020; Shi et al., 2020), although it can be used for assessing the immunogenicity of the upcoming vaccine candidates.

Importantly, the current study goes beyond other studies with SARS-CoV-2 and pigs since we tested a broader number of inoculation routes. However, none of them resulted in a productive infection in piglets. A significant outcome of this study was the evidence of seroconversion against the Spike glycoprotein at days 14 and $22 \mathrm{pi}$ and presence of neutralizing antibodies at day $22 \mathrm{pi}$ in pigs inoculated by parenteral routes (IM and IV). Considering the short duration of the experiment (22 days), such seroconversion emphasizes the potential interest of the pig to be used in immunogenicity studies for SARSCoV-2. In fact, the interest of swine as a suitable animal model for immunology, as well as physiology, pharmacology and surgery, applicable to human medicine is widely acknowledged (Rothkötter, 2009). 
In conclusion, the present study confirms that piglets are not a suitable animal model for COVID-19, but its potential usefulness as a model for immunogenicity in preclinical vaccine development studies deserves further investigation.

\section{ACKNOWLEDGEMENTS}

The authors thank the IRTA-CReSA staff for the care and handling of the animals. The authors also thank Nuria Navarro, Mónica Pérez, Rosa Valle and Marta Muñoz for their technical assistance. The CBIG Consortium (constituted by IRTA-CReSA, BSC, \& IrsiCaixa) is supported by Grifols pharmaceutical. The authors also acknowledge the crowdfunding initiative of https://www.yomecorono.com.

\section{DATA AVAILABILITY STATEMENT}

The data that support the findings of this study are openly available in GISAID (ID EPI_ISL_418268) Other data that support the findings of this study are mentioned in the manuscrit and/or available from the corresponding author upon reasonable request.

\section{ORCID}

Júlia Vergara-Alert (iD https://orcid.org/0000-0001-7484-444X Joaquim Segalés iD https://orcid.org/0000-0002-1539-7261

\section{REFERENCES}

Andersen, K. G., Rambaut, A., Lipkin, W. I., Holmes, E. C., \& Garry, R. F. (2020). The proximal origin of SARS-CoV-2. Nature Medicine, 26, 450-452. https://doi.org/10.1038/s41591-020-0820-9

Bao, L., Deng, W., Huang, B., Gao, H., Liu, J., Ren, L., Wei, Q., Yu, P., Xu, Y., Qi, F., Qu, Y., Li, F., Lv, Q., Wang, W., Xue, J., Gong, S., Liu, M., Wang, G., Wang, S., ... Qin, C. (2020). The pathogenicity of SARSCoV-2 in hACE2 transgenic mice. Nature, 583, 830-833. https://doi. org/10.1038/s41586-020-2312-y

Corman, V. M., Landt, O., Kaiser, M., Molenkamp, R., Meijer, A., Chu, D. K. W., Bleicker, T., Brünink, S., Schneider, J., Schmidt, M. L., Mulders, D. G. J. C., Haagmans, B. L., van der Veer, B., van den Brink, S., Wijsman, L., Goderski, G., Romette, J.-L., Ellis, J., Zambon, M., ... Drosten, C. (2020). Detection of 2019 novel coronavirus (2019$\mathrm{nCoV}$ ) by real-time RT-PCR. Eurosurveillance, 25, 2000045. https:// doi.org/10.2807/1560-7917.ES.2020.25.3.2000045

Damas, J., Hughes, G. M., Keough, K. C., Painter, C. A., Persky, N. S., Corbo, M., Hiller, M., Koepfli, K.-P., Pfenning, A. R., Zhao, H., Genereux, D. P., Swofford, R., Pollard, K. S., Ryder, O. A., Nweeia, M. T., LindbladToh, K., Teeling, E. C., Karlsson, E. K., \& Lewin, H. A. (2020). Broad host range of SARS-CoV-2 predicted by comparative and structural analysis of ACE2 in vertebrates. bioRxiv 2020.04.16.045302. https:// doi.org/10.1101/2020.04.16.045302

Garcia-Morante, B., Segalés, J., López-Soria, S., de Rozas, A. P., Maiti, H., Coll, T., \& Sibila, M. (2016). Induction of mycoplasmal pneumonia in experimentally infected pigs by means of different inoculation routes. Veterinary Research, 47, 54. https://doi.org/10.1186/s1356 7-016-0340-2

Halfmann, P. J., Hatta, M., Chiba, S., Maemura, T., Fan, S., Takeda, M., Kinoshita, N., Hattori, S., Sakai-Tagawa, Y., Iwatsuki-Horimoto, K., Imai, M., \& Kawaoka, Y. (2020). Transmission of SARS-CoV-2 in domestic cats. New England Journal of Medicine, 383, 592-594. https:// doi.org/10.1056/NEJMc2013400

Institut de Recerca de la sida (Irsicaixa) (2020). Detection of SARS-CoV-2 antibodies by elisa protocol v1 [Online]. Retrieved from http://www. irsicaixa.es/sites/default/files/detection_of_sars-cov-2_antibodies_ by_elisa_-_protocol_by_irsicaixa_protected.pdf

Kim, Y.-I., Kim, S.-G., Kim, S.-M., Kim, E.-H., Park, S.-J., Yu, K.-M., Chang, J.-H., Kim, E. J., Lee, S., Casel, M. A. B., Um, J., Song, M.-S., Jeong, H. W., Lai, V. D., Kim, Y., Chin, B. S., Park, J.-S., Chung, K.-H., Foo, S.-S., ... Choi, Y. K. (2020). Infection and Rapid Transmission of SARSCoV-2 in Ferrets. Cell Host \& Microbe, 27, 704-709.e2. https://doi. org/10.1016/j.chom.2020.03.023

Letko, M., Marzi, A., \& Munster, V. (2020). Functional assessment of cell entry and receptor usage for SARS-CoV-2 and other lineage B betacoronaviruses. Nat. Microbiol., 5, 562-569. https://doi. org/10.1038/s41564-020-0688-y

Meurens, F., Summerfield, A., Nauwynck, H., Saif, L., \& Gerdts, V. (2012). The pig: A model for human infectious diseases. Trends in Microbiology, 20, 50-57. https://doi.org/10.1016/j.tim.2011.11.002

Okba, N. M. A., Müller, M. A., Li, W., Wang, C., GeurtsvanKessel, C. H., Corman, V. M., Lamers, M. M., Sikkema, R. S., de Bruin, E., Chandler, F. D., Yazdanpanah, Y., Le Hingrat, Q., Descamps, D., Houhou-Fidouh, N., Reusken, C. B. E. M., Bosch, B.-J., Drosten, C., Koopmans, M. P. G., \& Haagmans, B. L. (2020). Severe acute respiratory syndrome coronavirus 2-specific antibody responses in coronavirus disease patients. Emerging Infectious Diseases, 26(7), 1478-1488. https://doi. org/10.3201/eid2607.200841

Qiu, Y., Zhao, Y.-B., Wang, Q., Li, J.-Y., Zhou, Z.-J., Liao, C.-H., \& Ge, X.-Y. (2020). Predicting the angiotensin converting enzyme 2 (ACE2) utilizing capability as the receptor of SARS-CoV-2. Microbes and Infection, 22, 221-225. https://doi.org/10.1016/j.micinf.2020.03.003

Rockx, B., Kuiken, T., Herfst, S., Bestebroer, T., Lamers, M. M., Oude Munnink, B. B., de Meulder, D., van Amerongen, G., van den Brand, J., Okba, N. M. A., Schipper, D., van Run, P., Leijten, L., Sikkema, R., Verschoor, E., Verstrepen, B., Bogers, W., Langermans, J., Drosten, C., ... Haagmans, B. L. (2020). Comparative pathogenesis of COVID19, MERS, and SARS in a nonhuman primate model. Science, 368 , 1012-1015. https://doi.org/10.1126/science.abb7314

Rodon, J., Noguera-Julian, M., Erkizia, I., Valencia, A., Guallar, V., Carrillo, J., Blanco, J., Segalés, J., Clotet, B., Vergara-Alert, J., \& Izquierdo-Useros, N. (2020). Search for SARS-CoV-2 inhibitors in currently approved drugs to tackle COVID-19 pandemia. bioRxiv 2020.04.23.055756. https://doi.org/10.1101/2020.04.23.055756

Rodon, J., Okba, N. M. A., Te, N., van Dieren, B., Bosch, B.-J., Bensaid, A., Segalés, J., Haagmans, B. L., \& Vergara-Alert, J. (2019). Blocking transmission of Middle East respiratory syndrome coronavirus (MERS-CoV) in llamas by vaccination with a recombinant spike protein. Emerging Microbes \& Infections, 8, 1593-1603. https://doi. org/10.1080/22221751.2019.1685912

Rothkötter, H.-J. (2009). Anatomical particularities of the porcine immune system-A physician's view. Developmental and Comparative Immunology, 33, 267-272. https://doi.org/10.1016/j.dci.2008.06.016

Saif, L. J., Pensaert, M. B., Sestak, K., Yeo, S. G., \& Jung, K. (2012). Coronaviruses. In J. J. Zimmerman, L. A. Karriker, A. Ramirez, K. J. Schwartz, \& G. W. Stevenson (Eds.), Diseases of Swine (pp. 501-524). Wiley-Blackwell.

Schlottau, K., Rissmann, M., Graaf, A., Schön, J., Sehl, J., Wylezich, C., Höper, D., Mettenleiter, T. C., Balkema-Buschmann, A., Harder, T., Grund, C., Hoffmann, D., Breithaupt, A., \& Beer, M. (2020). SARSCoV-2 in fruit bats, ferrets, pigs, and chickens: An experimental transmission study. The Lancet Microbe, 1, e218-e225. https://doi. org/10.1016/S2666-5247(20)30089-6

Shi, J., Wen, Z., Zhong, G., Yang, H., Wang, C., Huang, B., Liu, R., He, X., Shuai, L., Sun, Z., Zhao, Y., Liu, P., Liang, L., Cui, P., Wang, J., Zhang, X., Guan, Y., Tan, W., Wu, G., ... Bu, Z. (2020). Susceptibility of ferrets, cats, dogs, and other domesticated animals to SARS-coronavirus 2. Science, 368, 1016-1020. https://doi.org/10.1126/science.abb7015

Veljkovic, V., Vergara-Alert, J., Segalés, J., \& Paessler, S. (2020). Use of the informational spectrum methodology for rapid biological analysis 
of the novel coronavirus 2019-nCoV: Prediction of potential receptor, natural reservoir, tropism and therapeutic/vaccine target [version 3; peer review: 2 approved]. F1000Research, 9, 52. https://doi. org/10.12688/f1000research.22149.3

Vergara-Alert, J., van den Brand, J. M., Widagdo, W., Munoz, M., Raj, V. S., Schippr, D., Solanes, D., Cordon, I., Bensaid, A., Haagmans, B. L., \& Segales, J. (2017). Livestock susceptibility to infection with Middle East Respiratory Syndrome Coronavirus. Emerging Infectious Diseases, 23, 232-240. https://doi.org/10.3201/eid2302.161239

Vidal, A., Martín-Valls, G. E., Tello, M., Mateu, E., Martín, M., \& Darwich, L. (2019). Prevalence of enteric pathogens in diarrheic and non-diarrheic samples from pig farms with neonatal diarrhea in the North East of Spain. Veterinary Microbiology, 237, 108419. https://doi. org/10.1016/j.vetmic.2019.108419

World Health Organization (WHO) (2020). WHO Coronavirus Disease (COVID-19) Dashboard. Retrieved from https://covid19.who.int

Xiao, H., Nie, X.-T., Ji, X.-X., Yan, S., Zhu, B., \& Zhang, Y.-S. (2020). Establishing prokaryotic expression system of angiotensin-converting enzyme 2 (ACE2) gene in pigs. bioRxiv 2020.03.12.988634. https://doi.org/10.1101/2020.03.12.988634

Yu, P., Qi, F., Xu, Y., Li, F., Liu, P., Liu, J., Bao, L., Deng, W., Gao, H., Xiang, Z., Xiao, C., Lv, Q., Gong, S., Liu, J., Song, Z., Qu, Y., Xue, J., Wei, Q., Liu, M., ... Qin, C. (2020). Age-related rhesus macaque models of COVID-19. Animal Models and Experimental Medicine, 3, 93-97. https://doi.org/10.1002/ame2.12108
Zhou, P., Yang, X.-L., Wang, X.-G., Hu, B., Zhang, L., Zhang, W., Si, H.-R., Zhu, Y., Li, B., Huang, C.-L., Chen, H.-D., Chen, J., Luo, Y., Guo, H., Jiang, R.-D., Liu, M.-Q., Chen, Y., Shen, X.-R., Wang, X., ... Shi, Z.-L. (2020). A pneumonia outbreak associated with a new coronavirus of probable bat origin. Nature, 579, 270-273. https://doi.org/10.1038/ s41586-020-2012-7

\section{AUTHOR BIOGRAPHY}

Júlia Vergara-Alert is a veterinarian and researcher in the Animal Health Department (CReSA) at IRTA, Barcelona, Spain. Her primary research interest is in emerging diseases, mainly influenza viruses and coronaviruses.

How to cite this article: Vergara-Alert J, Rodon J, Carrillo J, et al. Pigs are not susceptible to SARS-CoV-2 infection but are a model for viral immunogenicity studies. Transbound Emerg Dis. 2020;00:1-5. https://doi.org/10.1111/tbed.13861 